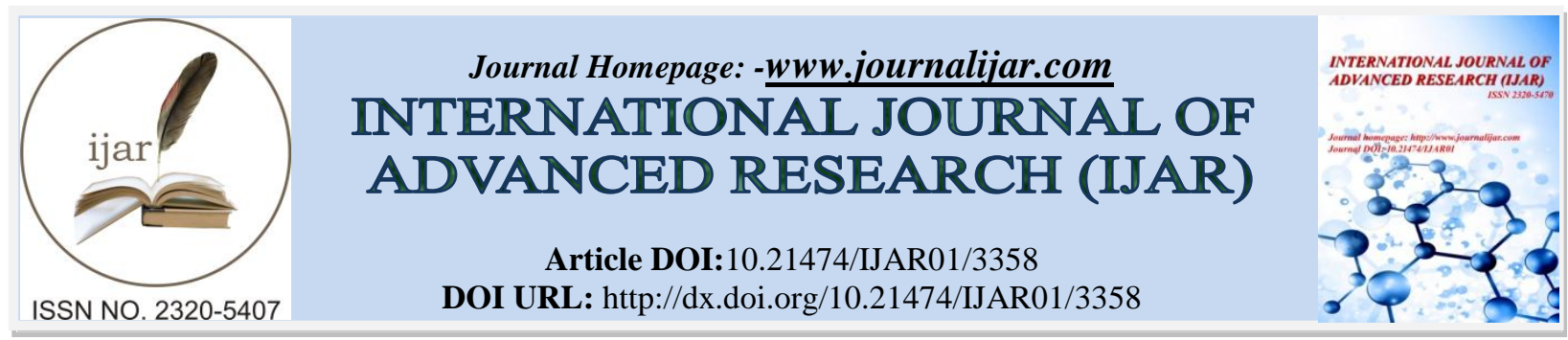

RESEARCH ARTICLE

\title{
REMOTE HOME MONITORING SYSTEM USING IOT DEVICES.
}

\section{Reenu Varghese ${ }^{1}$, Saju $A^{2}$ and Kuruvilla John ${ }^{2}$.}

1. PG Scholar, Department of Electronics and communication Engineering, Believers Church Caarmel Engineering College, Ranni-Perunad, Pathanamthitta,689711.

2. Associate Prof., Department of Electronics and communication Engineering, Believers Church Caarmel Engineering College, Ranni-Perunad, Pathanamthitta,689711.

\section{Manuscript Info}

Manuscript History

Received: 25 December 2016

Final Accepted: 12 January 2017

Published: February 2017

Key words: -

Internet of Things, Raspberry pi, Webcam

\begin{abstract}
IoT is expected to incorporate very large number of diverse devices ranging from simple networked sensors to large servers. For internetworking and communication among these devices, a seemingly large number of protocols have to be developed owing to the variety and difference in the hardware characteristics of each of these devices. Further, each IoT device should have a mechanism to identify and isolate the different kinds of messages in such a network. India faces a lot of security problems in the home or offices while we are not present. This paper aims to describe a IoT based motion detection mechanism system using low cost computing devices. Now a day's cost is most important factor so this project is reducing the cost for motion detection system. The objective of this work is to capture an image when any intruder motion is detected in prevented or secured area. Due to hardware limitations of Raspberry pi, motion detection is implemented by comparing the last two consecutive images saved locally. Any changes identified are notified to the user by uploading all the images from the point of identification, to a cloud server and via email.
\end{abstract}

Copy Right, IJAR, 2017, All rights reserved.

\section{Introduction: -}

The IoT means Internet of things. The internet of things interconnection between object or anything. The communication of IoT exchange the data over the network. Communication occurs without the machine to machine [1] interaction. In the new era, the all things are connected to internet of things. After the few years the internet of things interconnect with billions of devices. The IoT is used for many of the fields, Automobile, Agricultural, SecuritySurveillance, the internet of things is interconnecting to worldwide physical network. The main advantage of IoT is very low cost. The communication network has more complex and security issues are occurring.

Corresponding Author: -Reenu Varghese.

Address: -PG Scholar, Department of Electronics and communication Engineering, Believers Church

Caarmel Engineering College, Ranni-Perunad, Pathanamthitta,689711. 


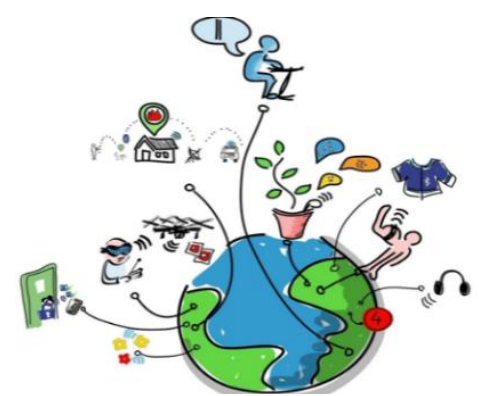

Fig 1:- Internet of Things

The main aim of this work is to the IoT concept using cloud server [2] to transfer the images. The motive behind the work is to develop a motion sensing (image processing) algorithm for IoT device considering the resource limitation and storage space limitations of such device, Further, the whole cost of the system is designed to be low. The figure 1 shows internet of things, it inter- connect with whole world. In 2020 the most of the world things are interconnect with IoT device.

\section{Motion Detection: -}

The existing systems use motion detection kits that usemotion sensors and Ethernet modules to send or receive data capturedby the sensors. This requires an external server to processthe data. The external server processes the data live streamed by the sensors. There is no local storage of the data in such systemsand if the cloud server is insecure, sensitive data can be leaked. Further, present secure systems use heavy data encryption algorithms to securely upload the data to the server. This approach is impractical in IoT in common IoT devices due to the hardware limitations.

Present systems also require large cloud storage to store such data and can use up the home internet bandwidth. The cost of such implementations are high. So, ordinary home users need an alternate method to reduce the cost of implementation using IoT based security hardware - without loss in efficiency and use ability. Further the requirement of existing systems to be always connected to the cloud server can handicap the functionality of the security of the intruder alert/notification function of the system since any problems in connectivity to the remote server or problems in the server can hinder communication between the sensors and the user.

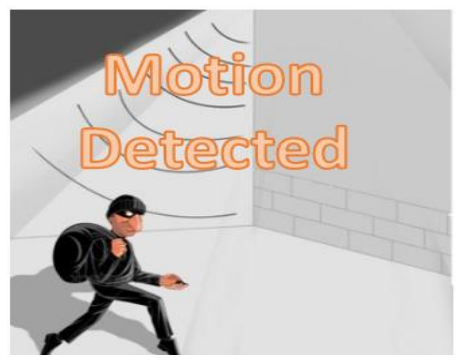

\section{Raspberry pi: -}

Fig 2: - Burglar detection based on frame comparison

Raspberry pi is a small size computer.it uses for low power processing. In this work we can use for latest version of raspberry pi, it known as model $3 \mathrm{~B}$. Raspberry pi model $3 \mathrm{~B}$ have the following features:

Operating system Raspbian, Fedora, Ubuntu MATE,Kali Linux,Debian

CPU $1.2 \mathrm{GHz} 64 / 32$-bit quad -core ARM cortex-A53

Memory

Storage

1GB LPDDR2 RAM at $900 \mathrm{MHz}$

Bluetooth

MicroSDHC

802.11.b/g/n Wireless LAN 


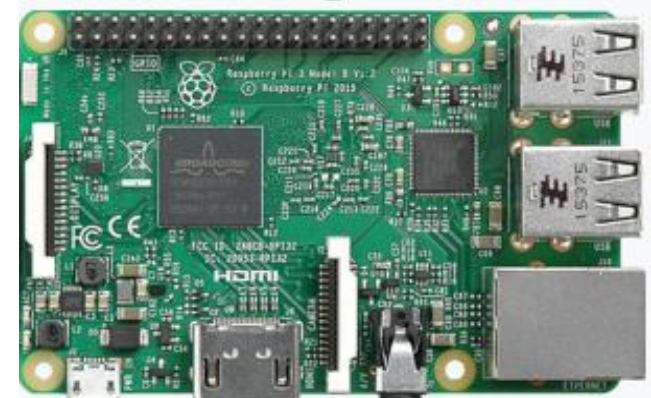

\section{Proposed Work: -}

Fig 3: -Raspberry Pi 3 Model B

This proposed method uses the motion detection using low cost computing devices. In this work we have used raspberry pi 3 model B to connect logic tech c 270 webcam to capture the intruding activities. The figure 3 shows the motion detection flow chart. Due to hardware limitations of Raspberry pi, motion detection is implemented by comparing the last two consecutive images saved locally. Any changes identified are notified to the user and all the images from the point of identification are uploaded to a cloud server and sent via email.

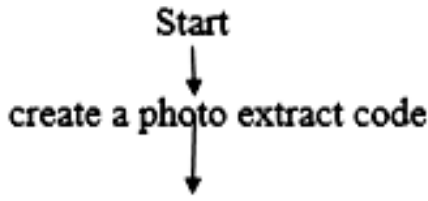

Compare to recent consecutive image<smiles>[13CH3]</smiles>

If there is considerable difference between two consecutive recent image

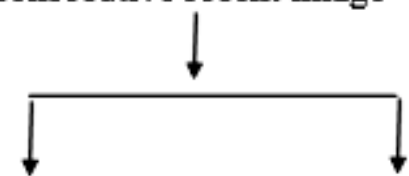

YES<smiles>C[13CH2]</smiles>

Notify the user

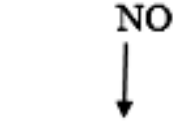

Take a pic raspberry pi

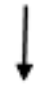

From the instant the photos are to differ they Will be store in the cloud

Fig 4: - Motion detection flow chart

In this work we can use the Gaussian Mixture Model algorithm [3][4]. This algorithm is generally used as a preprocessing stage in large image process project. The algorithm uses low resource. In addition to background subtraction, this also has ability to neglect small changes in the background such as changes in lighting condition etc which should not be perceived as motion. The segmentation approach also allows shadow [4] from the images to be removed.

Implementation part is Divides in four-part: -

- Representation of image as Gaussian Mixture Model.

- Background Subtraction.

- Image segmentation for motion detection. 
- Image segmentation for motion detection Minimum area threshold.

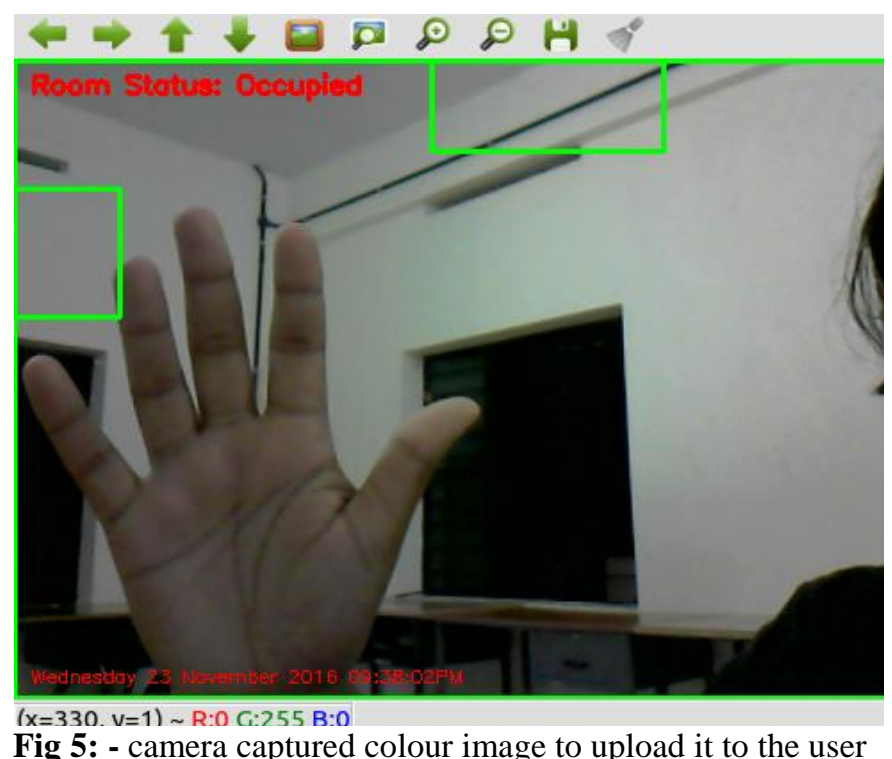

\section{Representation of image as Gaussian Mixture Model: -}

The probability density function representation of every naturally occurring signal, including images, show clusters of data. This means that some regions of an image has more data than other regions A Gaussian Mixture Model is the mathematical model of the image as the weighted sum of Gaussian component densities. For example, this image can be mathematically modelled as the sum of 3 Gaussian distribution functions, the coefficients of each of the function being the weights.

\section{Background Subtraction: -}

For motion detection, the background needs to be separated from the foreground. Background subtraction or Foreground Detection is used to extract an images foreground for further processing and object localisation.The pixels which remain static between frames are considered as the background and the pixels which are displaced from frame to frame are considered as the foreground. The absolute value of the pixel intensity differences difference between frames is obtained by a simple subtraction.

\section{Image segmentation for motion detection: -}

The goal of segmentation is to simplify and/or change the representation of an image. Our approach to image segmentation is to represent the background of the image as black (0) and the pixels where the motion is taking place in the image, called the foreground it represented in white (1). Finally, only regions where data is clustered are considered and unwanted data is removed.

\section{Image segmentation for motion detection Minimum area threshold: -}

If the contour area of the foreground segment is larger than our supplied minimum area called the threshold, motion can be considered to be detected. This method of comparing the foreground segment with that of the threshold area also helps to neglect any relatively small motion, like a paper moving in the breeze.

In this work, python script is used for motion detection. We can place camera in particular position and continuously monitor the area. If there is considerable difference between two consecutive [5] recent image the notification of motion detection is send to email and user phone as SMS. Through the website we can see the room from where ever we are and we can control raspberry pi 


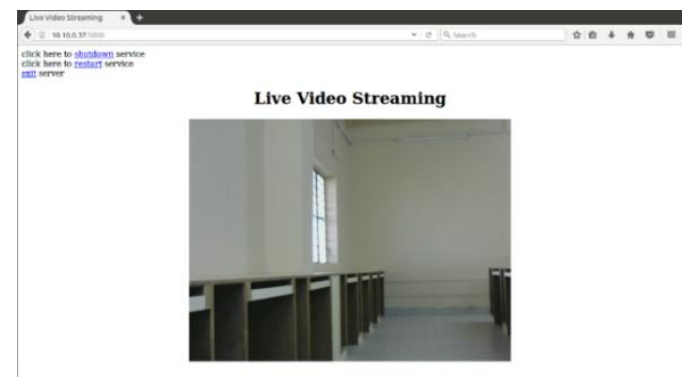

Fig 6: - Controlling raspberry pi remotely

\section{Conclusion: -}

The motion detection less space to store the data. Data privacy and safe communication is also considered to be achieved in the new communication system. The motive behind the project is to develop a motion sensing (image processing) algorithm for IoT device considering the resource limitation and storage space limitations of such device, Further, the whole cost of the system is designed to be low. Using this project, we can identify intruder motion. Which help to secure the area.

\section{References: -}

1. G. Anitha1, S.Juliya Fenita Devakumari2, G.Agasthiya3, Praveen Kumar,(2015) "An Internet of Things Approach for Motion Detection and Controlling Home Appliances Without Cloud Server" Student, Assistant Professor, Dept. of CSE, Anand Institute of Higher Technology, Rajiv Gandhi Salai, Kazhipattur, Chennai, Tamil,Nadu, India,vol.3,special issue 8,October 2015

2. Cheah Wai Zhao, Jayanand Jegatheesan,Son Chee Loon, (2015) "Exploring IOT Application Using Raspberry Pi” Quest International University Peraksf00391@qiup.edu.my,Volume 2, Issue 1, January - February (2015)

3. Zoran Zivkovic ,(2004)"Adaptive Gaussian Mixture Model for Background Subtraction" Intelligent and Autonomous Systems Group University of Amsterdam, The Netherlands email: zivkovic@science.uva.nlImproved.

4. P. KaewTraKulPong and R. Bowden (2001) "An Improved Adaptive Background Mixture Model for RealtimeTracking with Shadow Detection "Vision and Virtual Reality group, Department of Systems Engineering,BrunelUniversity,Middlesex,UB83PH,UK.Email:Pakorn.Kaewtrakulpong@ brunel.ac.ukRichard.B owden@brunel.ac.uk

5. Nishu Singla(2014) "Motion Detection Based on Frame Difference Method" Department of Computer Science Punjabi University, Patiala, Punjab. 